\title{
Clima organizacional: un diagnóstico en la empresa de motorepuestos Alarcón Perú
}

\section{Organizational atmosphere: a diagnosis in the Alarcón Peru motorcycle parts Company}

\author{
Susan Barboza ${ }^{1}$ (i), Ricardo Rivera²(i) y José Rafael ${ }^{3}$ (D)
}

\begin{abstract}
RESUMEN
La presente investigación tuvo como objetivo describir el clima organizacional en el cual se desempeñan los colaboradores de la empresa de motorepuestos Alarcón en el 2018, para lo cual se utilizó el tipo de metodología cuantitativa, nivel descriptivo, diseño no experimental y transversal; estando compuesta la muestra por 21 colaboradores. Para el acopio de los datos se utilizó como técnica la encuesta y como instrumento el cuestionario basado en las dimensiones propuestas por Mejía en el 2010 al cual se le adaptó las interrogantes de acuerdo a la realidad y giro de la empresa, este cuestionario estuvo compuesto por 45 preguntas. La información obtenida se analizó con el software estadístico SPSS 20.0, obteniendo tablas y figuras las cuales fueron analizadas e interpretadas; concluyendo que el Clima Laboral es desfavorable; asimismo se identificaron que predominan factores negativos como el liderazgo con $62 \%$, desarrollo personal y profesional con $62 \%$, identificación con la empresa $62 \%$, estabilidad laboral 57 $\%$ y condiciones ambientales $62 \%$; existiendo solo un factor positivo siendo este la remuneración con $62 \%$.
\end{abstract}

Palabras clave: clima laboral, percepción, satisfacción, insatisfacción, motivación.

\begin{abstract}
The objective of the present research was to describe the organizational atmosphere in which the employees of motorepuestos Alarcón's company are working in 2018, for which the type of quantitative methodology was used, descriptive level, non-experimental and transversal design; the sample is composed by 21 collaborators. For the collection of the data the survey was used as a technique and as a tool the questionnaire based on Mejia's dimensions 2010 to which the questions were adapted according to the reality and the company's turnaround, this questionnaire was composed of 45 questions. The information obtained was analyzed with the statistical software SPSS 20.0, obtaining charts and figures which were analyzed and interpreted; concluding that the Work Environment is unfavorable; It was also identified that negative factors predominate, such as leadership with $62 \%$, personal and professional development with $62 \%$, identification with the company $62 \%$, job stability $57 \%$ and environmental conditions $62 \%$. There is only one positive factor, being this the compensation with $62 \%$.
\end{abstract}

Keywords: work environment, perception, satisfaction, dissatisfaction, motivation.

DOI: https://doi.org/10.37787/pakamuros-unj.v9i21.161

Recibido: 24/11/2020. Aceptado: 07/02/2021

* Autor para correspondencia

1. Municipalidad Provincial de Jaén. Susan Barboza, Perú. Email: susan.barboza22@gmail.com

2. Dirección Sub Regional de Salud I Jaén. Ricardo Rivera, Perú, Email: ricardo.rivera.t22@gmail.com

3. La Fronterita, José Rafael, Perú. Email: jyd_rc@ @otmail.com 


\section{INTRODUCCIÓN}

El clima organizacional es un factor determinante en el logro de los objetivos de las instituciones, siendo un proceso complejo por la dinámica interna de las organizaciones, del entorno y de los factores humanos; asimismo, para generar equilibrio en el capital humano, en este caso de motorepuestos, la empresa requiere de procesos que midan habitualmente el clima organizacional con la finalidad de mantener colaboradores motivados, satisfechos y productivos (Chiavenato, 2017).

Así mismo, es un elemento importante y trascendente, permitiendo conocer la percepción de los individuos respecto a diversos factores que intervienen en el interior de una organización; al ser el ambiente de trabajo un factor primordial para el desarrollo de los colaboradores en su puesto de trabajo, existe la necesidad de un ambiente adecuado, lo cual permitirá un mejor desempeño, motivación y satisfacción en los colaboradores (Arroyo, 2010). Las organizaciones modernas tienen en claro que el activo más importante son sus trabajadores, al observarse que los individuos han dejado de ser considerados como máquinas que producen dinero. De acuerdo a lo referido, los gerentes deben proteger, retener y desarrollar este capital humano, propiciando en la organización un ambiente laboral adecuado en el cual se logren los objetivos individuales y organizacionales (Boguer, 2012).

De acuerdo a Wheter y Davis (2014) el clima laboral u organizacional no se asocia solo a las condiciones o estructura de la organización, sino también a factores psicosociales, problemas económicos, demográficos y sociales. Robbins y Judge (2013) sostienen que, si en la organización existe un clima de tipo autoritario, los colaboradores perciben y laboran en un ambiente tenso, con pocas recompensas, predomina el miedo, desconfianza y la comunicación es escasa con instrucciones específicas. Para Bruner (2007) el clima organizacional se encuentra constituido por características individuales y organizacionales constituyéndolo como su personalidad, siendo importante su diagnóstico y comprensión para la gerencia.

Estudios a nivel internacional se han desarrollado como es el de Chirinos, Meriño y Martínez (2018) quienes investigaron el clima organizacional en el emprendimiento sostenible, encontrando un nivel bajo de percepción debido a la escasa comunicación, inadecuadas decisiones, conflictos y falta de trabajo en equipo. Navarro (2017) buscó diagnosticar el clima organizacional, tras conocer la valoración que los trabajadores les otorgan diversas características de la empresa, el cual obtuvo como resultado la satisfacción en la mayoría las necesidades de los individuos, lo cual minimiza los comportamientos que perjudican a la compañía, percibiendo un clima organizacional satisfactorio. Chacón (2015) realizó una investigación para analizar el clima organizacional en la empresa CEM, tuvo como objetivo analizar los 
factores que afectan el clima organizacional, concluyó que el ambiente laboral es favorable, encontrándose deficiencias como la falta de reconocimiento e incentivos hacia los colaboradores.

Investigaciones nacionales similares como la de Mogollón y Ramírez (2019) al diagnosticar el clima laboral de los individuos discapacitados que trabajan en organizaciones de Piura, encontró un inadecuado y precario ambiente laboral basada en los resultados negativos de las diferentes dimensiones. Sandi (2017) investigó el clima organizacional que predomina en los trabajadores de Consulting Advisers S.A.C. con la Escala ECO, concluyendo que el clima organizacional es percibido como regular por los colaboradores, además se evidencian factores negativos como reconocimiento, relaciones, conflictos y desarrollo profesional. Belleza (2015) realizó el estudio sobre la percepción del clima organizacional en la Unidad de Gestión Educativa, el autor concluye que el clima organizacional en la institución es regular, resultado derivado de las dimensiones de Koy Decottis, presentando una menor valoración el reconocimiento.

En cuanto a los estudios regionales, no se han realizado en este tipo de organización, sin embargo, Quintana (2019) desarrolló estrategias de comunicación para mejorar el clima organizacional, encontrando un ambiente de trabajo adecuado y debilidades en los escasos equipos y herramientas, así como la baja remuneración. Roque y Vásquez (2018) tuvieron el objetivo de determinar si el clima organizacional influye en el funcionamiento de las instituciones, encontrándose resultados negativos en las dimensiones estructura, recompensa, relaciones e identidad, originando en un deficiente clima organizacional. El ambiente laboral es primordial para el desarrollo de los colaboradores en su puesto de trabajo, pues según diversos estudios realizados, indican que cuanto mejor se sienta el colaborador en un ambiente, mejor se desarrollará en su trabajo; asimismo, está presente en todas las organizaciones, sean privadas o públicas, por lo que es importante que los colaboradores se encuentren en las condiciones necesarias para que se desempeñen de forma eficaz y eficiente, permitiendo incrementar su satisfacción laboral y productividad para la organización (Palma, 2004).

Por la tanto, es importante para la empresa describir el clima organizacional el que se desempeñan los trabajadores, identificando factores percibidos como negativos, otorgando recomendaciones de mejora con el propósito de incrementar el grado de motivación de sus integrantes, traduciéndose en relaciones de satisfacción, ánimo, interés y colaboración (Gan, 2007).

La investigación ha sido desarrollada en base a información proporcionada por 21 colaboradores de la empresa de motorepuestos Alarcón de la ciudad de Jaén, provincia de Jaén, departamento de Cajamarca, Perú. 
En el contexto actual, las organizaciones deben adaptarse y adecuarse a los nuevos requerimientos para mantener su sostenibilidad, siendo uno de ellos el clima organizacional, señalado como aquellas características del ambiente laboral que son percibidas por los trabajadores y que predisponen su comportamiento. Chiavenato (2017), afirma que es el ambiente en el cual se desempeñan los individuos y asociado con el nivel de motivación de quienes lo componen. En cuanto a su función Gibson, Ivancevich y Donnelly (2011) sostiene que el clima organizacional delimita, genera identidad, proporciona compromiso, mantiene estabilidad y unidad, precisa las pautas y normas, además de guiar y controlar los comportamientos. Alles (2007, refiere que es el grupo de características de la personalidad, originadas por el comportamiento lo cual produce alto desempeño.

El clima organizacional es un término que se refiere específicamente a las propiedades motivacionales del ambiente laboral; es decir, a factores o aspectos de la empresa que estimulan o provocan distintos tipos de motivaciones en sus integrantes; presenta 9 dimensiones que permiten describirles en: comunicación, liderazgo, relaciones interpersonales, desarrollo personal y profesional, identificación con la institución, estabilidad laboral, remuneración, orientación a la calidad y condiciones ambientales. (Mejía,2010).

El objetivo de la investigación fue determinar la percepción del clima laboral en el cual se desempeñan los colaboradores de la empresa de motorepuestos Alarcón de la ciudad de Jaén en el 2018.

\section{MATERIALES Y MÉTODOS}

La investigación se desarrolló con un enfoque cuantitativo, basándose en la medición numérica y el análisis estadístico; el nivel de investigación fue descriptivo, diseño no experimental y corte transversal al no manipular la variable y acopiar la información en un momento único. La técnica para la recolección de datos fue la encuesta y como instrumento el cuestionario utilizando las dimensiones de Mejía, la cual estuvo compuesta por 45 interrogantes en escala de Licker adaptada al rubro de la organización, las mismas que fueron aplicadas a una muestra constituida por 21 colaboradores. Se utilizó el muestreo no probabilístico por conveniencia al tomar en consideración la totalidad de individuos que laboran en la empresa. Para el procesamiento de los datos se empleó el software estadístico SPSS versión 20.0, otorgándoles lectura e interpretación a las Figuras. El lugar donde se realizó la investigación fue el distrito de Jaén, empresa de motorepuestos Alarcón durante el periodo de marzo a octubre del 2018. 


\section{RESULTADOS}

Según la Figura 1, se logró determinar que el mayor porcentaje de colaboradores $52 \%$ opinan que el clima organizacional es desfavorable, mientras que el $19 \%$ de colaboradores considera que es favorable y $29 \%$ regular.

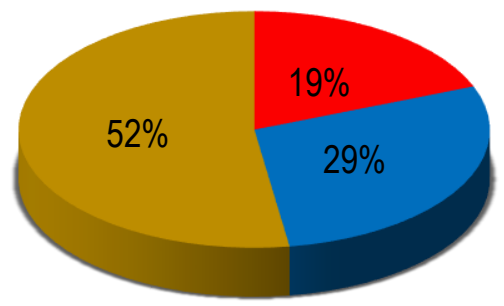

\section{- Favorable $\quad$ Regular}

Figura 1: Nivel de percepción del clima organizacional

La Figura 2, proporciona en su conjunto las diversas dimensiones utilizadas en la investigación, observándose que predominan factores negativos en la empresa tales como el Liderazgo con $62 \%$, Desarrollo Personal y Profesional con 62 \%, Identificación con la empresa 62 \%, Estabilidad Laboral $57 \%$, Condiciones Ambientales $62 \%$; solo existe un factor positivo, Remuneración con $62 \%$, y el resto de factores se perciben como regulares: Comunicación, Relaciones Interpersonales y Orientación a la Calidad.

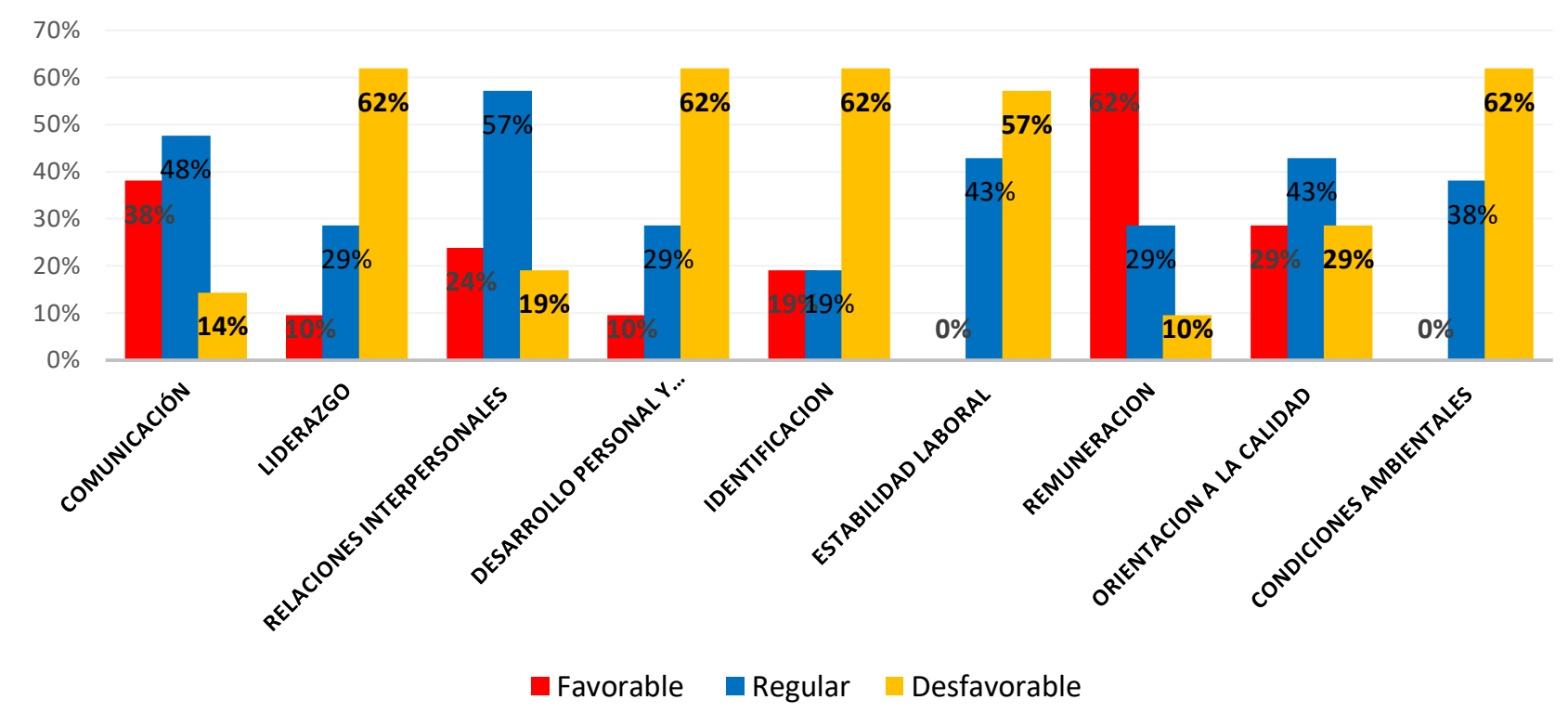

Figura 2: Niveles de percepción según dimensión del clima organizacional 


\section{DISCUSIÓN}

Para la dimensión comunicación, se realizaron 7 interrogantes, obteniendo como resultado en conjunto que el mayor porcentaje de colaboradores (48\%) señala como regular, el (38\%) como favorable y el menor porcentaje (14\%), identificando que la comunicación en la tienda de motorepuestos es regular, el resultado deriva de que cada trabajador realiza sus actividades en ocasiones individualmente sin solicitar apoyo; otros investigadores encontraron que los colaboradores realizan sus trabajos, en la mayoría de las oportunidades, sin solicitar apoyo por la escasa comunicación entre ellos (Sandi,2017; Belleza, 2015).

En cuanto a la dimensión liderazgo, se formularon 3 interrogantes, teniendo como resultado que el mayor porcentaje de trabajadores (62\%) sostiene que es desfavorable, el (29\%) como regular y el $10 \%$ señala que es favorable, poniéndose de manifiesto que el liderazgo es desfavorable en la empresa; este resultado es ocasionado por la poca comunicación y presencia del propietario, así como la demora en tomar decisiones para resolver los problemas y su condición de autocrático en sus acciones; hallazgos parecidos al Mogollón y Ramírez (2019) conjuntamente al de Roque y Vásquez (2018) al señalar que los directivos emiten órdenes sin tomar en consideración las sugerencias, de la misma manera manifiestan la escasa comunicación vertical y demora para la resolución de problemas.

Para la dimensión relaciones interpersonales, se desarrollaron 6 preguntas, teniendo como resultado al identificarlas que el mayor porcentaje de colaboradores (57\%) refieren como regular, el (24\%) como favorable y el menor porcentaje de colaboradores (19\%) señala que es desfavorable, conociendo que las relaciones interpersonales son regulares, hallazgo que mejoraría con la mejor interacción y comunicación entre compañeros de trabajo; resultados semejantes al de Chirinos, Meriño y Martínez (2018), Roque y Vásquez (2018) y Sandi (2017) al existir en sus organizaciones conflictos que generan negativas relaciones entre colaboradores, derivado de la escasa comunicación, interacción y apoyo entre ellos.

Referente a la dimensión desarrollo personal y profesional, se pusieron de manifiesto 6 interrogantes, obteniendo en conjunto que el mayor porcentaje (62\%) de trabajadores refieren que es desfavorable, el (29\%) que es regular y el menor porcentaje de colaboradores $(10 \%)$ sostienen que es favorable, identificándose que el desarrollo personal y profesional es desfavorable, concebido por el tamaño y organización de la empresa; existen pocas posibilidades de ascensos por el tamaño de la organización y escasos niveles jerárquicos, así como la alta rotación por temas políticos (Chacón, 2015; Mogollón,2019; Sandi, 2017)

Se visualiza que para la dimensión identificación, se realizaron 6 preguntas, las cuales al reunirlas se observa que el mayor porcentaje de empleados (62\%) sostiene que es desfavorable, mientras que el (19 
\%) están de acuerdo que es regular y favorable, lo que permite mencionar que la identificación es desfavorable; resultado negativo, debiendo ser considerado por las posibles consecuencias como la alta rotación de personal lo que perjudicaría la atención al cliente. La dimensión estabilidad laboral tuvo 5 interrogantes, las cuales al relacionarlas se obtiene que el mayor porcentaje (57\%) de los encuestados la identifican como desfavorable, mientras que el menor porcentaje de colaboradores de la empresa (43\%) manifiestan que es regular, por lo que se manifiesta que es desfavorable la estabilidad laboral, indicadores que resulta por no tener un contrato que les indique su estabilidad por un determinado tiempo; hallazgos equivalentes a Mogollón y Ramírez (2019) al establecer que es alta la rotación de los trabajadores sin un previo análisis o evaluación de su productividad, además de ser contratados por meses, aunado a las decisiones que se toman basadas en la política.

En lo referente a remuneración se desarrollaron 3 preguntas, de las cuales se puede señalar que el mayor porcentaje de trabajadores (62\%) sostiene que es favorable, el (29\%) que es regular y el $10 \%$ manifiesta que es desfavorable, identificando que la remuneración es favorable, lo consideran así al ser la empresa que ofrece mejor salario a sus colaboradores; conclusión parecida a Navarro (2017) al señalar los individuos que la remuneración es la adecuada y satisface sus necesidades, sin embargo no presentan similitud con las investigaciones de Chacón (2015) conjuntamente con Roque y Vásquez (2018) quienes ponen de manifiesto que el salario que ostentan los colaboradores no compensan el trabajo realizado.

Para la dimensión orientación a la calidad se formularon 4 preguntas, que al relacionarlas permitió reconocer que el mayor porcentaje de trabajadores (43\%) sostienen que es regular, mientras que el menor porcentaje (29\%) señalan que es favorable y desfavorable, lo que motiva a sostener que la orientación a la calidad es regular, lo refieren así puesto que no se han dejado de lado los procesos de mejora continua y calidad de servicio (Sandi, 2017; Belleza, 2015).

En cuanto a la dimensión condiciones ambientales, se propusieron 5 interrogantes, las que en conjunto ponen de manifiesto que el mayor porcentaje de encuestados (62\%) refieren que es desfavorable, mientras que el menor porcentaje (38\%) manifiesta que es regular, señalando que las condiciones ambientales es desfavorable, lo cual pone de manifiesto que el ambiente donde se desempeñan no es el adecuado, perjudicando el correcto desarrollo de las funciones de los colaboradores; resultados homogéneos son el de Mogollón y Ramírez (2019) al señalar los colaboradores que realizan sus funciones en condiciones inestables, Quintana (2019) al no otorgarles las herramientas y equipos necesarios, así también con Roque y Vásquez (2018) al no contar con la infraestructura adecuada para realizar sus actividades. 
Al realizar el análisis general de las dimensiones del clima organizacional, se observa que el mayor porcentaje de colaboradores (52\%) señala que es desfavorable, el (29\%) regular, y en menor porcentaje (19\%) sostiene que es favorable; por lo tanto, se determina que el clima organizacional es desfavorable. Estos hallazgos son favorables cuando satisface las necesidades personales de los integrantes elevando la moral, mientras que es desfavorable cuando frustra esas necesidades; los resultados de la presente investigación muestran que el clima es desfavorable, pues al analizar sus diversas dimensiones, el mayor porcentaje de estas no cubre las necesidades de los colaboradores (Chirinos,2018; Chiavenato, 2017).

Lo referido por Robbins y Judge (2013) ratifican lo acontecido en el estudio, al encontrar como factor negativo el liderazgo, pues los autores manifiestan que, bajo un clima de tipo autoritario, los individuos perciben y trabajan en una atmósfera tensa, con muy pocas recompensas, predominando el miedo y la desconfianza, así también la comunicación sólo existe en forma directa con instrucciones específicas, manifestaciones que perciben y sienten los colaboradores de la organización analizada.

\section{CONCLUSIONES}

El clima organizacional se logra conceptualizar como las características internas que generan en los individuos la percepción del ambiente donde desarrollan sus actividades, si es favorable producirá efectos positivos en su desempeño y comportamiento, caso contrario perjudicará a la organización al no lograr los objetivos propuestos. Mediante la investigación, se logró determinar que la percepción del personal en cuanto al clima organizacional es desfavorable (52\%).

Al desarrollar las diferentes dimensiones, se pudo identificar que, para los colaboradores de la empresa, solamente existe un factor percibido como positivo, siendo la Remuneración (62 \%), pues según señalan los encuestados, la empresa de motorepuestos es la que mejor paga en el medio.

Se logró identificar la presencia de dimensiones percibidas como negativas, encontrándose entre ellas el Liderazgo (62 \%), Desarrollo Personal y Profesional (62 \%), Identificación con la empresa (62 \%), Estabilidad Laboral (57 \%) y Condiciones Ambientales (62\%); los demás factores como Comunicación (48\%), Relaciones Interpersonales (57\%) y Orientación a la Calidad (43\%) fueron considerados por los trabajadores de motorepuestos Alarcón como regulares. 


\section{REFERENCIAS BIBLIOGRÁFICAS}

Alles, M. (2007). Desarrollo del talento humano: basado en competencias. $3^{\mathrm{a}}$ ed. Buenos Aires: Granica

Arroyo, J. (2010). Relaciones entre el clima organizacional y satisfacción laboral en una empresa de servicios. (Tesis post grado). Universidad Pontifica Comillas. España.

Baguer, A. (2012). Alerta!: descubre de forma sencilla y práctica los problemas graves de tu empresa, sus vías. 7ed. Colombia: Ediciones Díaz de Santos

Belleza, C. (2015). Percepción del clima organizacional en la Unidad de Gestión Educativa Local No 08 Cañete, 2015. (Tesis pre grado). Universidad César Vallejo. Recuperado de https://repositorio.ucv.edu.pe/handle/20.500.12692/5363

Brunet, L. (2007). El clima de trabajo en las organizaciones. México: Trillas.

Chacón, V. (2015). Análisis del clima organizacional de la empresa representaciones CEM, ubicada en Chiquimula. (Tesis pre grado). Universidad Rafael Landivar de Guatemala.

Chiavenato, I. (2017).Administración de Recursos Humanos. El capital humano de las organizaciones. 10ed. México: Mc.Graw-Hill.

Chirinos, A., Meriño, C., y Martínez, M. (2018). El clima organizacional en el emprendimiento sostenible. Revista EAN. 84. 43-61. Recuperado de http://www.scielo.org.co/pdf/ean/n84/01208160-ean-84-00043.pdf

Gan, F. (2007). Manual de Recursos Humanos 1ed. México: Prentice Hall.

Gibson, J., Ivancevich, H., y Donelly, J. (2011). Las organizaciones: comportamiento, estructura y procesos, 13ed. México: Mc Graw-Hill.

Mejía, M. (2010). Manual para realizar Diagnostico del Clima Laboral. Ministerio de Salud Pública y Asistencia Social, Guatemala

Mogollón, G., y Ramírez, A. (2019). Clima laboral de las personas con discapacidad en las organizaciones de la ciudad de Piura 2019. Tzhoecoen, (12)2, 191-199. Recuperado de http://revistas.uss.edu.pe/index.php/tzh/article/view/1257/1141

Navarro, M. (2017). Diagnóstico del clima organizacional para trabajadores contratados por outsourcing. Caso: Bendita Paleta. (Tesis de maestría). Instituto Politécnico Nacional. Recuperado de https://docplayer.es/58294645-Instituto-politecnico-nacional-unidad-profesionalinterdisciplinaria-de-ingenieria-y-ciencias-sociales-y-administrativas.html

Palma, S. (2004). Escala de clima laboral (CL-SPC) Manual. Lima: Editora y Comercializadora CARTOLAN EIRL. 
Quintana, R. (2019). Plan de estrategias en comunicación asertiva para mejorar la relación entre el clima organizacional y el desempeño laboral en la I.E.. $\mathrm{N}^{\circ}$ 16020, del distrito de Jaén, provincia Jaén 2019. (Tesis pre grado). Universidad Señor de Sipán. Recuperado de https://cutt.ly/4khrulh

Robbins, S. y Judge, T. (2013). Comportamiento Organizacional. 15a ed. México: Pearson educación.

Roque, R., y Vásquez, V. (2018). Influencia del clima organizacional en el funcionamiento de las instituciones educativas de la red educativa “Túpac Amaru”, San Ignacio - Cajamarca. (Tesis de maestría). Universidad César Vallejo. Recuperada de https://cutt.ly/okhqJ4q

Sandi, G. (2017). Clima Organizacional predominante en los colaboradores de la empresa Consulting Advisers S.A.C. Lima-2017. (Tesis pre grado). Universidad Inca Garcilaso de la Vega. Recuperado de http://repositorio.uigv.edu.pe/handle/20.500.11818/2251

Wheter, W., y Davis K. (2014). Administración de Recursos Humanos, Gestión del Capital Humano 7ed. México: Mc Graw-Hill. 